\title{
NeXo AGUA - ENERGía: ANÁlisis DEL FLUJO HÍDRICO DEL PROYECTO HidROELÉCTRICO Coca Codo Sinclair
}

\author{
ThE WATER-ENERGY NEXUS: ANALYSIS OF \\ THE WATER FLOW OF THE COCA CODO \\ Sinclair Hydroelectric ProjeCt
}

\author{
Sebastián Jiménez-Mendoza ${ }^{1}$, Francisco Terneus-Páez ${ }^{1,2, *}$
}

\section{Resumen}

Este artículo analiza la interrelación existente entre el agua y la energía, considerando el flujo hídrico del Proyecto Hidroeléctrico Coca Codo Sinclair. Investiga el caso de este proyecto emblemático, donde los usos consuntivos del agua disminuirían el caudal de entrada al túnel de conducción, arriesgando con esto su capacidad de generación eléctrica. Se utiliza para ello una investigación de tipo bibliográfica. Se concluye que el proyecto Chalpi Grande y las fases siguientes de ríos orientales; y los proyectos de riego Cayambe-Pedro Moncayo y de agua potable PesilloImbabura afectarían el caudal de entrada hasta en un $11 \%$ y con ello su producción de energía, con lo cual queda en evidencia la necesidad de planificar el aprovechamiento de estos recursos considerando su nexo.

Palabras clave: Nexo agua - energía, Proyecto Hidroeléctrico Coca Codo Sinclair, uso consuntivo del agua.

\begin{abstract}
This article analyzes the interrelation between water and energy, taking as a case the analysis of the water flow of the Coca Codo Sinclair Hydroelectric Project. Investigate the case of this emblematic project, where the water uses of consumption would decrease the inflow to the driving tunnel, which would risk its power generation capacity. Bibliographic research is used for this purpose. It is concluded that the Chalpi Grande project and the following phases of the Rios Orientales; and the Cayambe Pedro Moncayo irrigation projects and the Pesillo Imbabura potable water would affect the flow of inputs of the hydroelectric project by up to $11 \%$ and, therefore, their energy production, demonstrating the need to plan the use of these resources considering their nexus.
\end{abstract}

Keywords: Water-energy nexus, Coca Codo Sinclair Hydroelectric Project, consumptive use of water.

\footnotetext{
$\overline{1, *}$ Departamento de Energía y Mecánica, Universidad de las Fuerzas Armadas ESPE, Sangolquí, Ecuador (D) http://orcid.org/0000-0001-7031-5954

${ }^{2}$ Programa de Doctorado en Gestión Tecnológica - Escuela Politécnica Nacional, Quito, Ecuador. Autor para correspondencia cfterneus@espe.edu.ec (D) http://orcid.org/0000-0001-5372-0288
}

Recibido: 02-10-2018, aprobado tras revisión: 30-11-2018

Forma sugerida de citación: Jiménez-Mendoza, S. y Terneus-Páez, F. (2019). «Nexo agua - energía: Análisis del flujo hídrico del Proyecto Hidroeléctrico Coca Codo Sinclair ». Ingenius. N. ${ }^{\circ}$ 21, (enero-junio). pp. 53-62. DOI: https://doi.org/10.17163/ings.n21.2019.05. 


\section{Introducción}

El nexo entre el agua-energía-alimentos ha entrado en debate desde la Conferencia de Bonn del 2011 en la cual se recomendó que estos recursos se consideren de manera integradora, y centrándose en asegurar que las interdependencias entre estos estén explícitamente identificadas en la toma de decisiones [1]. Tres años más tarde, durante la conferencia del Global Water Security 83 Sanitation Partnership (GWSP), las comunidades de investigación y políticas de todo el mundo emitieron un llamado para desarrollar estrategias que aborden un enfoque de nexo completo [2]. Con la actual tasa de crecimiento de la población mundial, el sector agrícola enfrenta el desafío de duplicar la producción de alimentos para 2050 [3]. Alrededor del $71 \%$ de las extracciones de agua del mundo se atribuyen al sector agrícola [4]. Para el 2050 se necesitará un aumento del $55 \%$ en la demanda mundial de agua para abordar el aumento de la generación de electricidad y uso doméstico, se proyecta que más del $40 \%$ de la población mundial vivirá bajo estrés hídrico severo [5]. Sin embargo, muy pocos autores han abordado la cuestión de cómo traducir este concepto del nexo agua-energía-alimentos, que es principalmente teórico, en enfoques prácticos de evaluación. Albrecht et al. [6] afirman que, a pesar del enfoque conceptual prometedor, el uso del nexo agua-energía-alimentos como herramienta analítica para evaluar sistemáticamente las interconexiones de estos recursos ha sido limitado. Middleton et al. [7] menciona que el nexo agua-energía-alimento aún no se ha integrado en la práctica. Del mismo modo, Leck et al. [8] solicita la aplicación práctica del nexo agua-energía-alimento en las futuras investigaciones científicas.

Denise Lofman et al. [9] consideran que el nexo entre el agua y la energía presentará dificultades para equilibrar las necesidades de los usuarios y proteger recursos valiosos a la vez, esto referente a temas agrícolas, industriales y residenciales. Pittock Jamie et al. [10] muestran como el nexo entre el suministro de energía hidroeléctrica y el suministro de alimento influye considerablemente en la cuenca aportante de agua. Según Fisher, J. et al. [11] el nexo agua-energía para la generación de electricidad trae problemas más severos como la contaminación y la emisión de $\mathrm{CO}_{2}$. Lubega et al. [12] afirma que es posible medir el nexo agua-energía mediante modelos entre la energía eléctrica y el consumo municipal de agua. Varias tendencias actuales aumentan aún más la urgencia de abordar el nexo agua-energía de una manera integrada y proactiva. En primer lugar, el cambio climático ya ha comenzado a afectar los patrones de precipitación y temperatura. En segundo lugar, el crecimiento de la población, y las tendencias de migración regional indican que es probable que aumenten habitantes en zonas áridas. Por último, la introducción de nuevas tecnologías en los ámbitos de la energía y el agua podría cambiar las demandas de estos recursos [13].

La Agencia Internacional de Energía [14] proyecta para el año 2040, un aumento del uso consuntivo del agua en el mundo en casi un $60 \%$, afectando a hidroeléctricas de la misma zona cuya extracción de agua aumentará en menos del $2 \%$. Debido al crecimiento poblacional y los cambios en la dieta, el consumo de alimentos está aumentando en casi todas las regiones del mundo. Se espera que para el año 2050 sea necesario producir 200 millones de toneladas de carne y 1 billón adicional de toneladas de cereal al año para poder satisfacer la creciente demanda de alimentos. Por ello la agricultura es responsable del $90 \%$ del uso consuntivo del agua [15].

Debido a estos problemas mundiales, el país se ve en la necesidad de abordar y planificar el aprovechamiento de sus recursos hídricos considerando su nexo. La Ley de Recursos Hídricos del Ecuador menciona en el artículo 30: «El Estado y sus instituciones en el ámbito de sus competencias son los responsables de la gestión integrada de los recursos hídricos por cuenca hidrográfica. En consecuencia, son los obligados a regular los usos, el aprovechamiento del agua y las acciones para preservarla en cantidad y calidad mediante un manejo sustentable a partir de normas técnicas y parámetros de calidad» [16].

Por otra parte, en el Ecuador, la Constitución del 2008, en el Art. 313 determina: «El Estado se reserva el derecho de administrar, regular, controlar y gestionar los sectores estratégicos; se consideran sectores estratégicos la energía en todas sus formas...» [17]. Este artículo tiene como objetivo analizar el caso del Proyecto Hidroeléctrico Coca Codo Sinclair (PHCCS); como ejemplo del nexo existente entre el agua y la energía; donde los usos consuntivos del agua disminuirían el caudal de entrada al túnel de conducción, arriesgando con esto su capacidad de generación eléctrica de este proyecto emblemático. A continuación, en la sección 1.1, se muestra la síntesis de la historia del PHCCS, en la sección 2 se analiza como metodología, los informes de factibilidad del PHCCS y los proyectos que hacen uso consuntivo del agua; en la sección 3 se analiza como dichos proyectos afectarían al caudal de ingreso del PHCCS y, finalmente, en la sección 4 se ofrece unas conclusiones evidenciando la necesidad de planificar el nexo agua-energía de manera integrada.

\subsection{El Proyecto Hidroeléctrico Coca Codo Sin- clair}

El PHCCS es una obra que fue calificada como emblemática por el Gobierno ecuatoriano, se construyó en el origen del cauce del río Coca en la provincia de Napo [18], toma su nombre por el geólogo norteamericano Joseph Sinclair, quien al recorrer el río Coca en el Oriente ecuatoriano, identificó una curva marcada, 
que luego fue bautizada por la población del sector como Codo Sinclair. Este investigador afirmó que este lugar tenía la posibilidad de ser utilizada para generar energía eléctrica [19].

El PHCCS, fue uno de los proyectos más importantes del Plan Nacional de Electrificación, en la cuenca de los ríos Quijos y Coca, durante los años setenta y ochenta, siendo el Instituto Ecuatoriano de Electrificación (INECEL), la empresa encargada de realizar los estudios. En 1976 se realizaron dos, el de prefactibilidad por la empresa brasileña Hidroservice y el de la capacidad total de aprovechamiento por empresas consultoras italianas Electroconsult y Rodio, la empresa belga Tractionel y las empresas nacionales Ingeconsult, Inelin, Astec y Caminos y Canales [20].

Entre abril de 1990 y junio de 1992 para la optimización de la alternativa seleccionada, se realizó el diseño de factibilidad en dos etapas continuas que generarían 432 (MW) y 427 (MW) de potencia respectivamente sumando un total de 859 (MW) de potencia. Este estudio incluía modificaciones del proyecto debido al terremoto que hubo cerca del volcán Reventador en el mes de marzo de 1987, el cual cambió notablemente la fisonomía del terreno. En el 2007 el Estado modificó dicho estudio y fue rediseñado hasta alcanzar una potencia de 1500 (MW) [18].

El PHCCS fue anunciado el 15 de enero del 2007 y fue considerado de alto interés nacional, siendo incluido en el Plan Maestro de Electrificación. En ese año, las empresas encargadas de su gestión fueron dos; el primer trimestre fue el Consejo Nacional de Electricidad (Conelec) y en julio fue el Ministerio de Electricidad y Energías Renovables (MEER). Sin embargo, cabe anotar que como operadora del proyecto había sido designada, en septiembre del 2007, la empresa del Estado ecuatoriano Termopichincha, que más adelante pasa a conformar con la empresa argentina ENARSA el consorcio Coca Sinclair S. A. [20].

En el 2008 los estudios fueron aprobados por el Conelec. La Compañía Hidroeléctrica Coca Codo Sinclair S. A. de Quito, encargó a la Compañía Consultora ELC-Electroconsult de Milán, Italia los estudios de rediseño conceptual para alcanzar 1500 (MW). En el 2009 ELC-Electroconsult presenta el estudio de factibilidad final de 1500 (MW). La Compañía Hidroeléctrica Coca Codo Sinclair S. A. de Quito contrata a la empresa SINOHYDRO para el desarrollo de ingeniería y es esta la que inicia su construcción [20]. Después de seis años, el 18 de noviembre de 2016 se inaugura el PHCCS [21].

Tras finalizar la construcción, las principales obras que conforman el PHCCS son la obra de captación que cuenta con una presa de 31,8 (m) de altura máxima, vertedero con una altura del azud de $13,5(\mathrm{~m})$ y ancho neto de $160(\mathrm{~m})$, desarenador de 8 cámaras. El túnel de conducción con una longitud de 24,8 (km), 9,10 (m) de diámetro de excavación y un diámetro inte- rior de $8,20(\mathrm{~m})$, diámetro de diseño de $222\left(\mathrm{~m}^{3} / \mathrm{s}\right)$. El embalse compensador conformado por una presa de enrocado con cara de hormigón de $58(\mathrm{~m})$ de altura, para crear un embalse útil de $800000\left(\mathrm{~m}^{3}\right)$. Las tuberías de presión son dos y conducen el agua desde el embalse compensador hasta la casa de máquinas, en hormigón y con revestimiento de acero en su tramo final, de $1400(\mathrm{~m})$ de longitud y tienen un diámetro interno de 5,8 y $5,2(\mathrm{~m})$ respectivamente, el caudal de diseño es de $139,25\left(\mathrm{~m}^{3} / \mathrm{s}\right)$ cada una. La casa de máquinas es una caverna excavada en roca de $26 \times 46,8 \times 219,5(\mathrm{~m})$ donde se ubican 8 turbinas tipo Pelton de eje vertical con 6 inyectores cada una y una potencia unitaria de 187,5 (MW), que turbina las aguas del río Coca, que se forma a partir de la unión de los ríos Quijos y Salado como se observa en la Figura 1 [22].

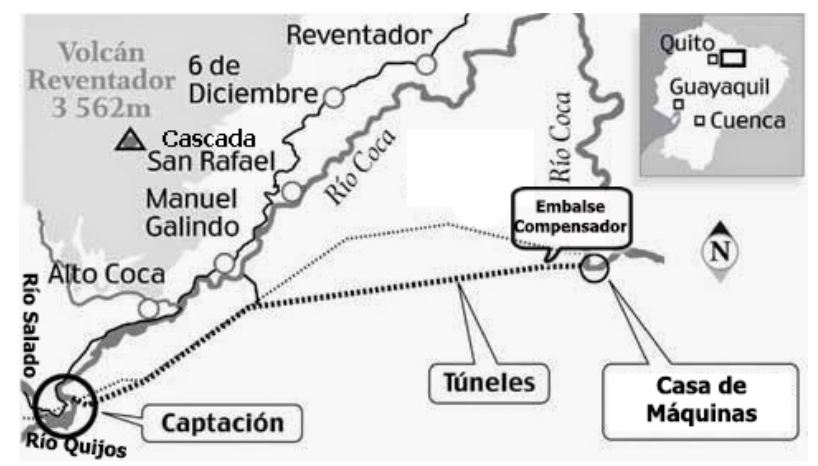

Figura 1. Ubicación del PHCCS (adaptado de [23]).

Utilizando la ecuación general (1) que rige la potencia instalada de una central hidroeléctrica, denominada también potencia útil nominal, se calcula el caudal necesario para generar la potencia de 1500 (MW), detallándose la misma a continuación [24].

$$
P i=\eta t \times \eta g \times \eta t r \times \lambda \times Q \times H
$$

donde:

$P i=$ Potencia útil nominal $(\mathrm{W})$

$Q=$ Caudal que entra en la tubería de presión

$\left(\mathrm{m}^{3} / \mathrm{s}\right)$

$H=$ Altura neta nominal $(\mathrm{m})$

$\eta t=$ Eficiencia de la turbina Pelton

$\eta g=$ Eficiencia del generador

$\eta t r=$ eficiencia del transformador

A continuación, se muestran los datos obtenidos del anexo $f$ del informe de factibilidad del PHCCS realizado por ELC-Electroconsult [25].

$$
\begin{aligned}
& H=604,1(\mathrm{~m}) \\
& P=1500(\mathrm{MW}) \\
& \eta t=91 \% \\
& \eta g=97,52 \% \\
& \eta t r=99,5 \%
\end{aligned}
$$


Reemplazando los datos en la ecuación (1), se tiene:

$$
\begin{gathered}
P i=\eta t \times \eta g \times \eta t r \times 9,81 \times Q \times H \times 1000 \\
Q=\frac{P i}{\eta t \times \eta g \times \eta t r \times 9,81 \times H \times 1000} \\
Q=\frac{1500000000}{0,91 \times 0,9752 \times 0,995 \times 9,81 \times 604,1 \times 1000} \\
Q=286,6\left(\mathrm{~m}^{3} / \mathrm{s}\right)
\end{gathered}
$$

Dado que el PHCCS es una central de pasada con regulación diaria [25], para generar 1500 (MW), el caudal turbinado, es decir, el caudal que debe ingresar a las dos tuberías de presión es de $286,6\left(\mathrm{~m}^{3} / \mathrm{s}\right)$, es decir, 143,3 ( $\left.\mathrm{m}^{3} / \mathrm{s}\right)$ en cada una. Según Synohidro Corporation, el PHCCS solo puede generar esta potencia 4 horas diarias, sin embargo, el caudal de diseño de las tuberías de presión es de 139,25 ( $\left.\mathrm{m}^{3} / \mathrm{s}\right)$, es decir, $278,5\left(\mathrm{~m}^{3} / \mathrm{s}\right)$ las dos [25].

En el 2017, tras un año de operación, el PHCCS ha producido el 66,7 \% de la energía esperada. Entre enero y diciembre, la central, aportó al sistema nacional interconectado un total de 5838 (GWh), una cifra por debajo de la generación media proyectada, que es de 8734 (GWh) [21].

\section{Materiales y métodos}

La investigación presentada es de tipo bibliográfica con un alcance descriptivo. Se analiza el estudio de factibilidad que presentó INECEL en el año 1992 donde se observa el comportamiento histórico del caudal del río Coca, el estudio de factibilidad que presentó ELCElectroconsult que muestra el rediseño del estudio de Inecel. Además, se revisan los proyectos que afectarían el caudal que ingresa al PHCCS debido a los usos consuntivos de agua.

\subsection{Estudios de factibilidad}

A continuación, se va a analizar los dos informes de factibilidad del PHCCS, el primero corresponde al estudio que realizó Inecel aprobado en el año de 1992 y el segundo corresponde al estudio que realizó ELCElectroconsult aprobado en el año 2009.

\subsubsection{Estudio de factibilidad de 1992 del PHCCS}

El estudio de factibilidad fue realizado por Inecel. Para calcular los caudales del proyecto, se partió de datos históricos del río Coca y la cascada San Rafael, el lapso comprendido fue desde 1972 a 1987 para la cascada San Rafael y desde 1972 a 1987 para el río Coca en el sector El Salado [18]. Dado que este estudio se hizo en una misma estación fluvial y durante un lapso de quince a veinte periodos que abarcan años secos, normales y húmedos, esta metodología es ampliamente aceptada [26]. En la Figura 2, se observa los caudales medios mensuales registrados en la estación del río Coca en el sector El Salado durante los lapsos de tiempo antes señalados. La curva de duración general de caudales diarios en el sitio El Salado elaborada por INECEL se ilustra en la Figura 3 [19].

\section{Caudales afluentes al PHCCS}

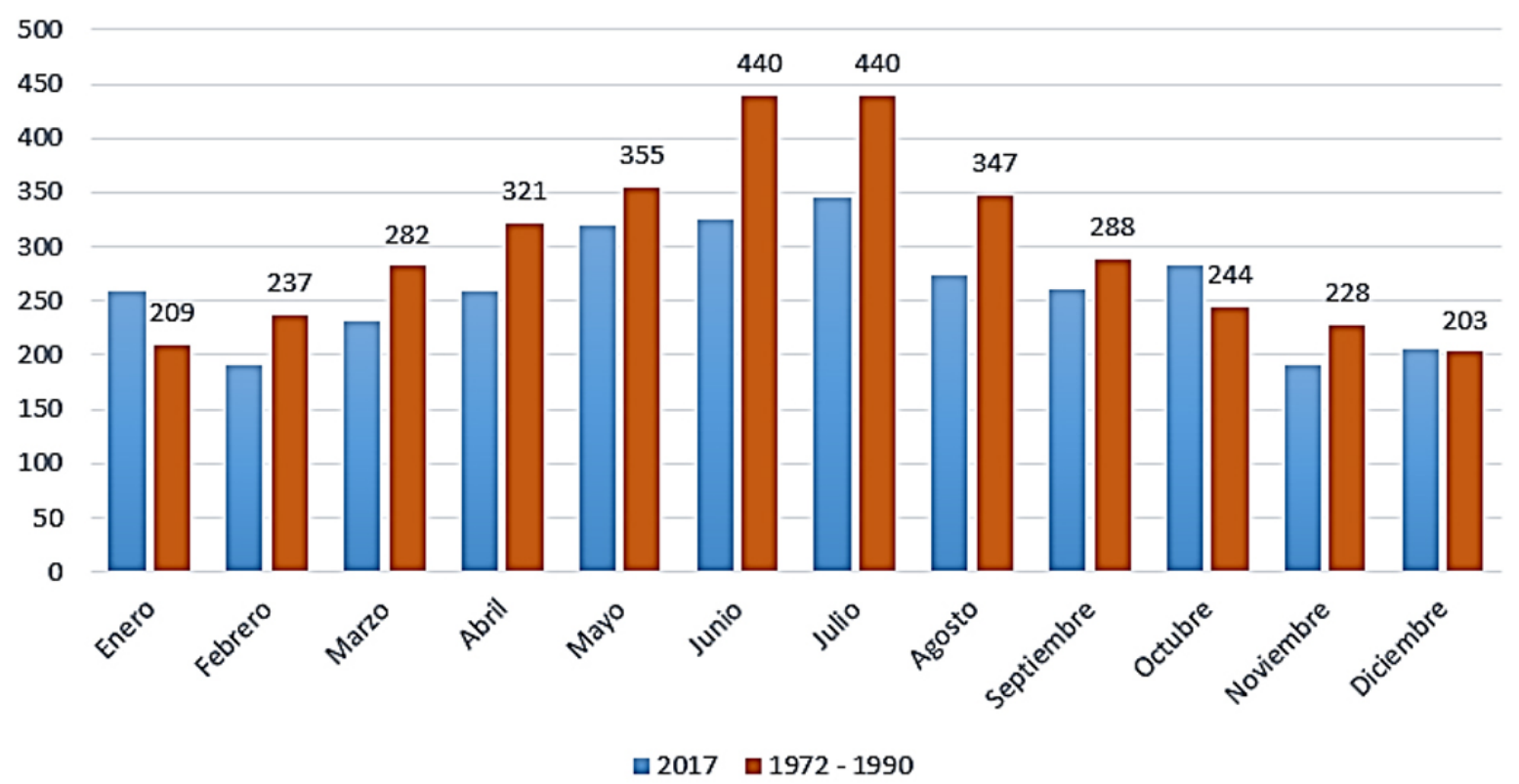

Figura 2. Comparación entre los caudales del 1972-1990 y del 2017. 
De esta manera, Inecel determina que el caudal promedio del río Coca en el sector El Salado es de 292 $\left(\mathrm{m}^{3} / \mathrm{s}\right)$ descontando $3\left(\mathrm{~m}^{3} / \mathrm{s}\right)$ que eran tomados por el acueducto Papallacta-Quito, lo que corresponde a una contribución especifica superior a los $80\left(\mathrm{l} / \mathrm{s} / \mathrm{km}^{2}\right)$. El caudal firme diario garantizado en un $90 \%$ del tiempo es de $127\left(\mathrm{~m}^{3} / \mathrm{s}\right)[18]$.

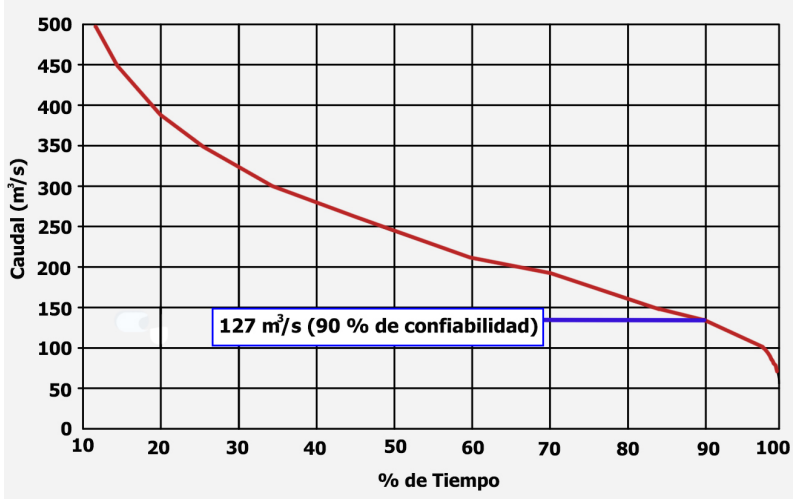

Figura 3. Curva de duración general de caudales diarios en el sitio El Salado (adaptado de [19]).

La empresa Inecel decidió que el caudal se debía captar en 2 etapas, la primera debía ser de $63,5\left(\mathrm{~m}^{3} / \mathrm{s}\right)$, al cual se tenían que añadir otros $63,5\left(\mathrm{~m}^{3} / \mathrm{s}\right)$ en segunda alcanzando así en total $127\left(\mathrm{~m}^{3} / \mathrm{s}\right)$. Tanto en primera etapa como en la segunda el factor de planta se había mantenido igual a $0,8[22]$.

\subsubsection{Estudio de factibilidad actual del PHCCS}

El estudio de factibilidad actual del PHCCS fue realizado por ELC-Electroconsult, y se basó en el estudio hidrológico que realizó Inecel en el año 1992 donde se registra el caudal histórico del río Coca [22].

ELC-Electroconsult señaló que para alcanzar los 1500 MW instalados en la casa de máquinas, manteniendo las mismas pérdidas del estudio de factibilidad en las obras de caída, se requeriría un caudal máximo de $278,5\left(\mathrm{~m}^{3} / \mathrm{s}\right)$ en la tubería de presión, lo que corresponde a un caudal entrante en el embalse compensador de $222,7\left(\mathrm{~m}^{3} / \mathrm{s}\right)$. Restando $0,7\left(\mathrm{~m}^{3} / \mathrm{s}\right)$ del caudal promedio de la quebrada Granadilla, se obtiene el valor de $222,0\left(\mathrm{~m}^{3} / \mathrm{s}\right)$. Por lo tanto, el caudal a ser derivado en el sitio El Salado a la presa y que transitaría en el túnel de conducción es de 222,0 $\left(\mathrm{m}^{3} / \mathrm{s}\right)$ [22].

Para obtener un caudal máximo de $278,5\left(\mathrm{~m}^{3} / \mathrm{s}\right)$ en la tubería de presión manteniendo el factor de planta $(0,8)$, se requirió aumentar el volumen útil del embalse compensador de $460000\left(\mathrm{~m}^{3}\right)$ a $800000\left(\mathrm{~m}^{3}\right)$ manteniendo siempre los mismos valores de niveles mínimo y máximo, es decir, 1229,50 y 1216,00 (m s. n. m.) respectivamente [22].

Por otro lado, CENACE dispone información sobre el caudal afluente al embalse compensador del PHCCS que se muestran en la Tabla 1. En la Figura 2 se puede evidenciar una disminución de los caudales del año 2017 con los históricos de los obtenidos de 1972-1990.

Cabe considerar el cambio significativo en la fisionomía del sector, debido principalmente a la construcción de la vía Valle de Quijos-Lago Agrio, la cual fue entregada en 1972 y fomentó la colonización del sector con la consecuente transformación de la masa boscosa en pastizales y la explotación maderera, lo cual seguramente afectó las condiciones climáticas de esta cuenca hidrológica y el caudal de sus ríos [27]. Estos últimos se ven afectados también por los usos consuntivos del agua.

Tabla 1. Caudal afluente al embalse compensador del PHCCS [21]

\begin{tabular}{ccc}
\hline \multirow{2}{*}{ Meses } & \multicolumn{2}{c}{ Caudal $\left(\mathbf{m}^{3} / \mathbf{s}\right)$} \\
\cline { 2 - 3 } & $\mathbf{2 0 1 6}$ & $\mathbf{2 0 1 7}$ \\
\hline Enero & - & 258,75 \\
\hline Febrero & - & 190,21 \\
\hline Marzo & - & 230,37 \\
\hline Abril & - & 258,11 \\
\hline Mayo & 697,9 & 320,35 \\
\hline Junio & 707,38 & 324,84 \\
\hline Julio & 560,6 & 344,57 \\
\hline Agosto & 506,23 & 272,94 \\
\hline Septiembre & 394,34 & 259,8 \\
\hline Octubre & 298,5 & 283,55 \\
\hline Noviembre & 148,03 & 190,05 \\
\hline Diciembre & 135,89 & 205,16 \\
\hline
\end{tabular}

\subsection{Usos consuntivos del agua}

La Ley Orgánica de Recursos Hídricos, Usos y Aprovechamiento del Agua, define al uso consuntivo como aquel en el que el líquido vital, una vez utilizado, no se devuelve al medio donde se ha captado, ni de la misma manera que se ha extraído [16]. Estos tipos de usos se pueden identificar en cuatro proyectos; uno ya existente, dos en construcción y uno programado como muestra la Figura 4, que van a captar o captan el agua que conforman el caudal que ingresa al PHCCS, estos son los siguientes:

\section{Existente:}

- Proyecto Papallacta de la Empresa Pública Metropolitana de Agua Potable y Saneamiento del Distrito Metropolitano de Quito (EPMAPS). 
En construcción:

- Proyecto Chalpi Grande o etapa uno del Proyecto Ríos Orientales (PRO) de la EPMAPS.

- Proyecto de riego Cayambe-Pedro Moncayo y agua potable Pesillo-Imbabura.

Programado:

- Etapas futuras del Proyecto Ríos Orientales (PRO) de la EPMAPS.

A continuación, se describirán a cada uno de ellos.

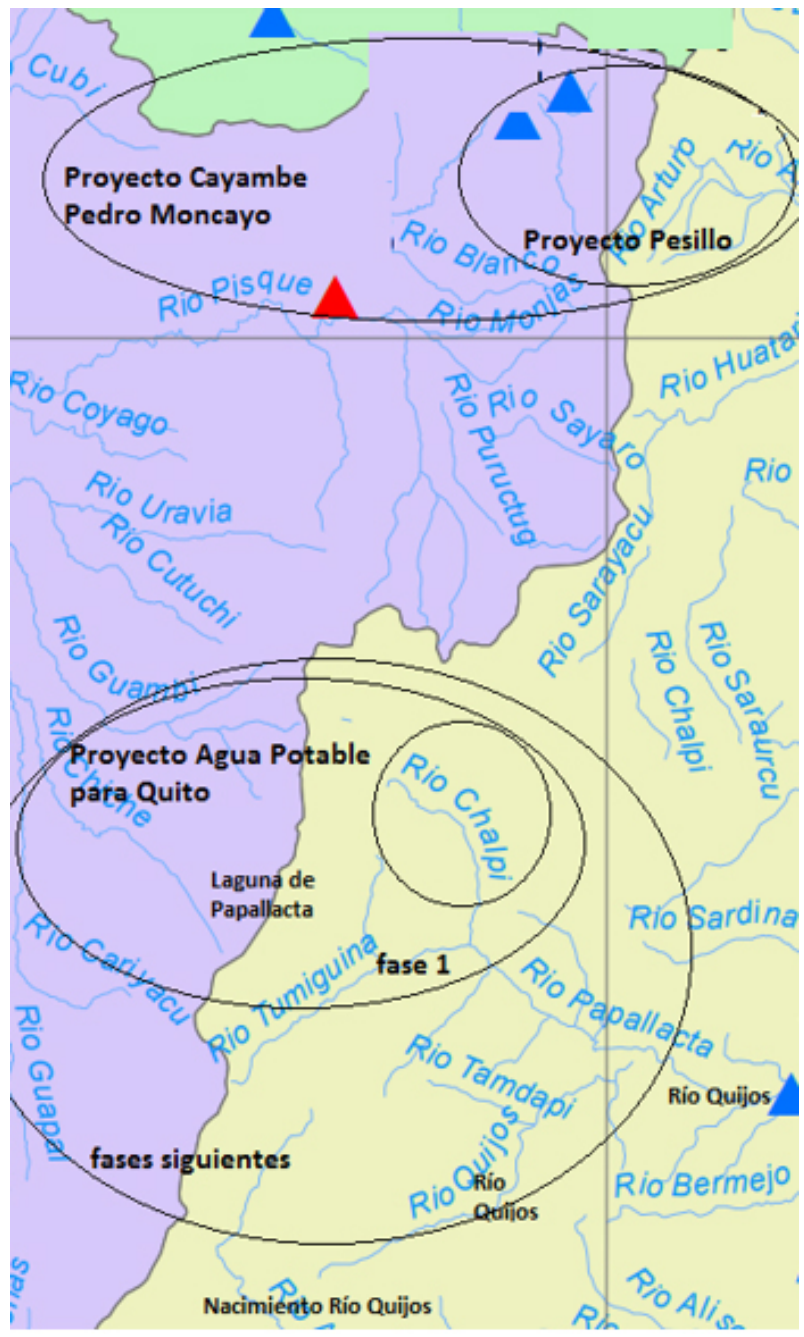

Figura 4. Ubicación de proyectos en construcción [28].

\subsubsection{Proyecto Papallacta}

Es un proyecto inaugurado en 1990 por la Empresa Metropolitana de Alcantarillado y Agua Potable de Quito, EPMAPS, consiste en el abastecimiento de agua potable para la ciudad de Quito, en la provincia de Pichincha. En sentencia del 22 de septiembre de 1987 mediante la concesión con número de proceso 1503, la empresa obtuvo el permiso de captar el caudal de los ríos Papallacta con un caudal de $1,70\left(\mathrm{~m}^{3} / \mathrm{s}\right)$; Chalpi Grande con un caudal de $3,20\left(\mathrm{~m}^{3} / \mathrm{s}\right)$; Tuminguina con un caudal de $2,20\left(\mathrm{~m}^{3} / \mathrm{s}\right)$ y Blanco Chico con un caudal de $0,90\left(\mathrm{~m}^{3} / \mathrm{s}\right)[29,30]$.

Para los estudios de factibilidad del PHCCS se tomó en cuenta el valor de $3\left(\mathrm{~m}^{3} / \mathrm{s}\right)$, sin embargo, la concesión otorgada a EPMAPS fue de $8\left(\mathrm{~m}^{3} / \mathrm{s}\right)$ [30].

\subsubsection{Proyecto Ríos Orientales (PRO)}

En la década de los setenta se analizó la creciente demanda de agua potable en la ciudad de Quito, la cual exigía la implementación de nuevos proyectos, además de reducir las pérdidas no contabilizadas y el consumo per cápita. EPMAPS decidió diseñar algunos proyectos, de ellos, el más importante es el Proyecto Ríos Orientales (PRO), el cual abastecería de agua al Distrito Metropolitano de Quito y a sus 22 parroquias rurales hasta después del año 2055 mediante la captación, conducción a gravedad, tratamiento y transmisión de 31 ríos. El PRO aprovecharía aguas de las cuencas hidrográficas de los ríos Valle Vicioso, Antisana, Cosanga, Quijos y Papallacta, situadas sobre todo en los cantones Quijos y Archidona de la provincia de Napo [31]. El 16 de enero de 2002, mediante la concesión con número de proceso 296-96-CTD [29], EPMAPS obtuvo el permiso, por parte del entonces Consejo Nacional de Recursos Hídricos (CNRH), hoy Senagua, de captar las aguas de los ríos que alimentarán al proyecto, los cuales se resumen en la Tabla 2 [31].

Tabla 2. Caudales concedidos por el CNRH a la EPMAPS en enero de 2002 [31]

\begin{tabular}{cc}
\hline Río & Caudal $\left(\mathbf{m}^{3} / \mathbf{s}\right)$ \\
\hline Río Valle Vicioso & 5,01 \\
Río Tolda & 0,74 \\
Río Chuzalongo & 0,3 \\
Río Bajo & 0,16 \\
Río Antisana & 4,49 \\
Río Javas & 0,71 \\
Río Cosanga & 1,13 \\
Río Quijos Sur & 2,14 \\
Río Quijos Norte & 1,36 \\
Río Blanco Grande & 1,19 \\
\hline Total & $\mathbf{1 7 , 2 3}$
\end{tabular}

Por aspectos técnicos, económicos-financieros y ambientales, la construcción del Proyecto Ríos Orientales (PRO) se ejecutaría por etapas como se muestra en la Figura 5 [32]. La primera etapa usaría la concesión del año de 1987, mientras que la segunda etapa y las etapas futuras usarían la concesión del año 2002, la cual se resumió en la Tabla 2. 


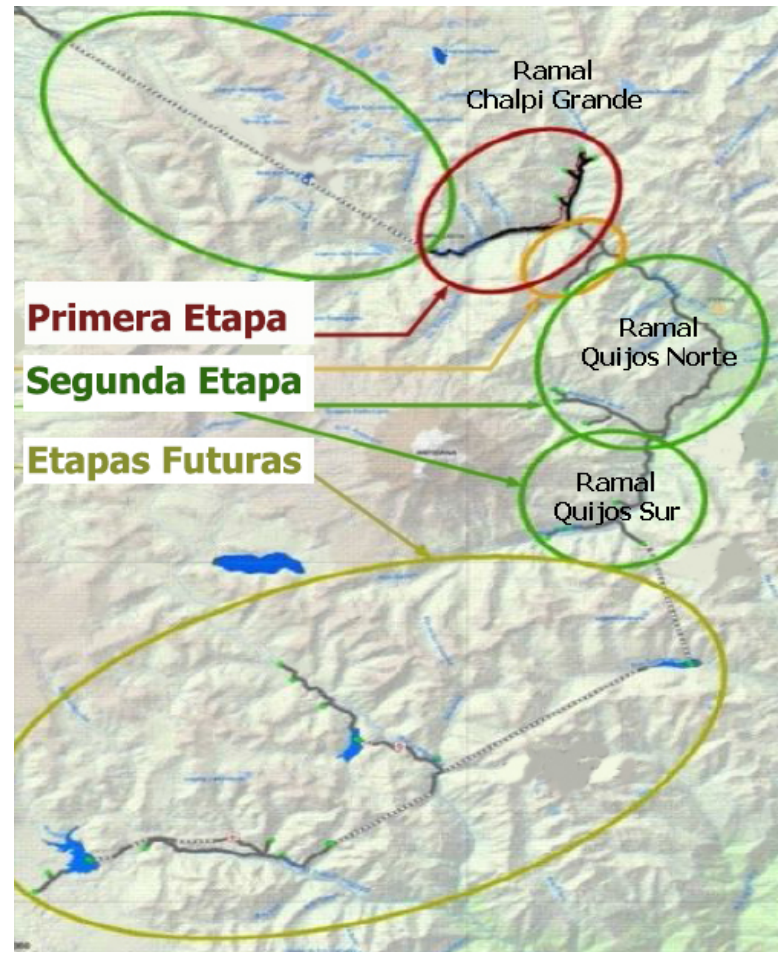

Figura 5. Etapas del Proyecto Ríos Orientales (adaptado de [33]).

La primera etapa: Ramal Chalpi GrandePapallacta comprende un canal que captará el caudal de $2,21\left(\mathrm{~m}^{3} / \mathrm{s}\right)$ de los afluentes: Chalpi A $(1,23$ $\left.\mathrm{m}^{3} / \mathrm{s}\right)$, Encantado $\left(0,64 \mathrm{~m}^{3} / \mathrm{s}\right)$, Chalpi B $\left(0,27 \mathrm{~m}^{3} / \mathrm{s}\right)$ y Chalpi $\mathrm{C}\left(0,07 \mathrm{~m}^{3} / \mathrm{s}\right)$ que conforman el río Chalpi Grande como se muestra en la Figura 6, y lo transvasa al reservorio en Papallacta [33].

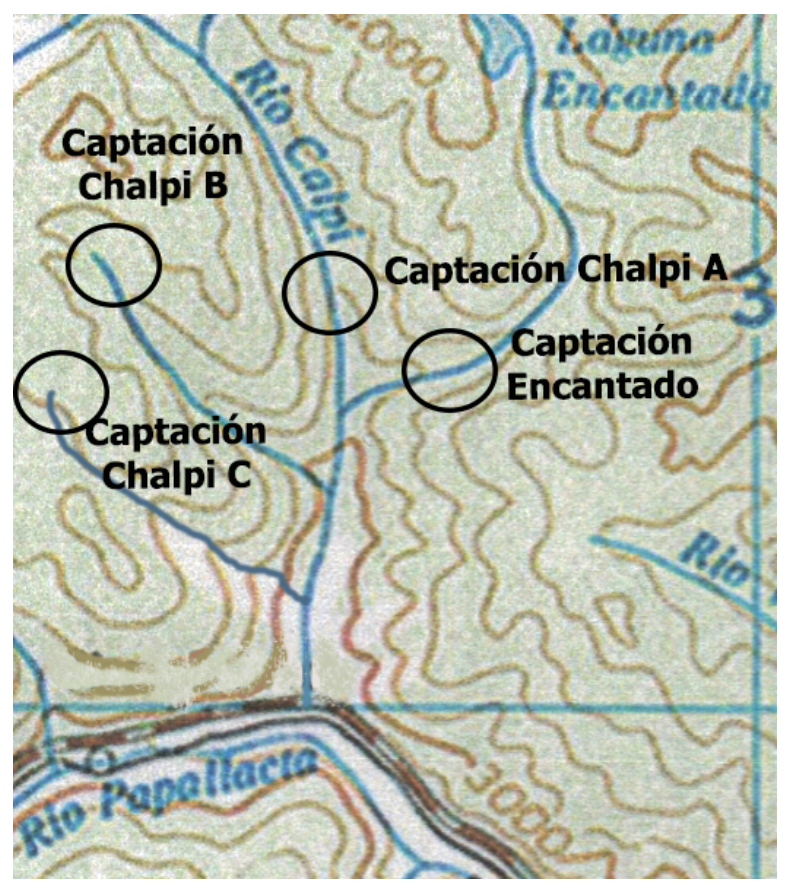

Figura 6. Etapa 1 del PRO (adaptado de [34]).
En julio del 2018, el gerente de la EPMAPS señaló que el proyecto registra un avance del $26 \%$ y su culminación será en el 2021 [35].

La segunda etapa: Ramal Quijos-PapallactaPaluguillo empezará en el año 2040 y tiene previsto captar un caudal total de 4,691 ( $\left.\mathrm{m}^{3} / \mathrm{s}\right)$ de los ríos Quijos Norte, Tablón, Cristal, Pucalpa, Azufrado, Semiond, Quijos Sur y Blanco Grande [36].

Las etapas futuras empezarán en el año 2041 y tiene previsto obtener un caudal de los ríos Cosanga, Antisana, Valle Viscoso y sus afluentes [32].

\subsubsection{Proyecto de riego Cayambe-Pedro Mon- cayo y agua potable Pesillo-Imbabura}

El Proyecto de riego Cayambe-Pedro Moncayo y agua potable Pesillo-Imbabura tiene por objetivo captar las aguas de los ríos Arturo, Boquerón, San Pedro, San Jerónimo y Montoneras a través de túneles de aducción que conectan al río Arturo con el Boquerón, al río Boquerón con el San Pedro y al San Pedro con La Rápida, como muestra la Figura 7 [37]. En el primer proceso, el 220-96, la Resolución de la Agencia de Aguas de Quito, dictada el 15 de abril de 1999, favoreció al Gobierno Provincial de Pichincha, concediéndole el derecho de uso y aprovechamiento de las aguas de los ríos Azuela, Arturo, Boquerón y San Pedro, con un caudal total de $3,325\left(\mathrm{~m}^{3} / \mathrm{s}\right)[38]$.

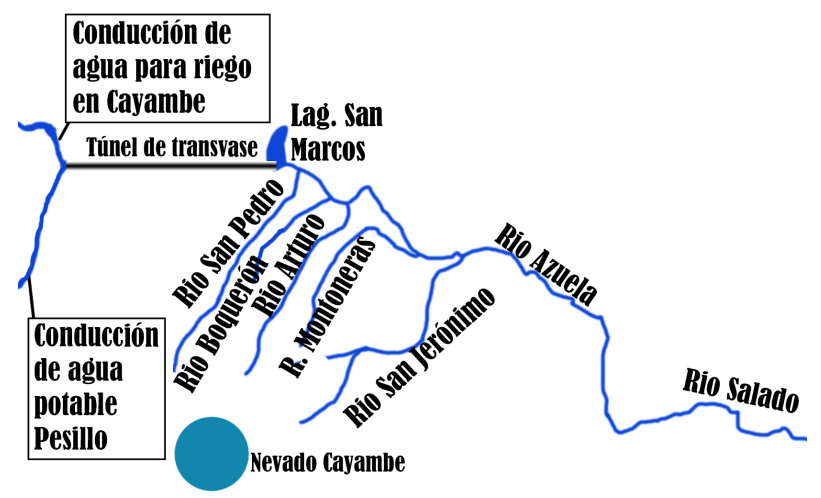

Figura 7. Ubicación del Proyecto Cayambe-Pedro Moncayo y Pesillo-Imbabura (adaptado de [33]).

En el segundo proceso, el 1375-00, la Agencia de Aguas de Quito concede al Gobierno Provincial de Pichincha el derecho de aprovechamiento de las aguas que provienen de los ríos San Jerónimo, Montoneras, La Chimba, y sus afluentes. De estos ríos, los que afectan al caudal del río Salado que alimenta al PHCCS, son los dos primeros, con un caudal de $0,24\left(\mathrm{~m}^{3} / \mathrm{s}\right)$ y $0,08\left(\mathrm{~m}^{3} / \mathrm{s}\right)$ respectivamente. Asimismo, la Agencia de Aguas de Quito concede a favor del Gobierno Provincial de Imbabura, las aguas que provienen de los ríos Montoneras con un caudal de $0,1\left(\mathrm{~m}^{3} / \mathrm{s}\right)$ y San Jerónimo con un caudal de 0,31 ( $\left.\mathrm{m}^{3} / \mathrm{s}\right)$ [39]. Dichos caudales, 
sumados al de la anterior concesión, que también desembocan en El Salado, de los ríos Arturo, Boquerón y San Pedro, totaliza $4,06\left(\mathrm{~m}^{3} / \mathrm{s}\right)[39]$.

El 13 de diciembre del 2017 el director del proyecto de riego Cayambe-Pedro Moncayo y agua potable Pesillo-Imbabura, informó que el proyecto registra un avance del 95,60\% [37].

\section{Resultados y discusión}

A continuación, en la Figura 8 se recopila los eventos pasados y futuros que afectarían al caudal del PHCCS.

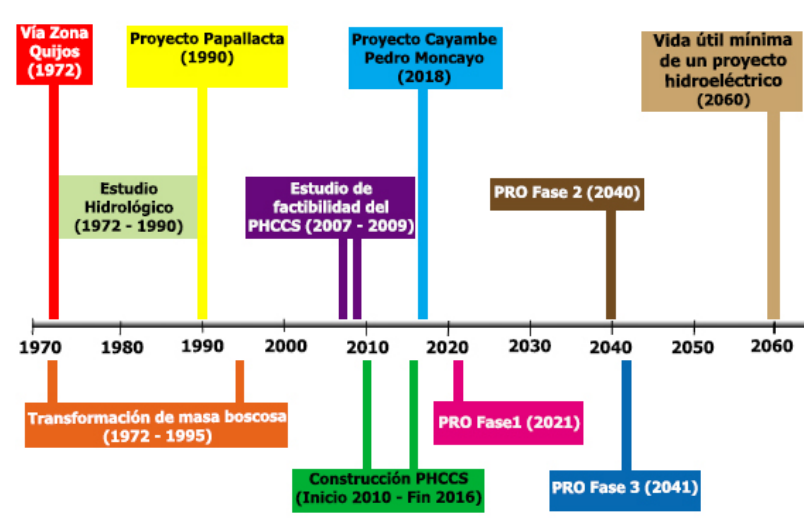

Figura 8. Línea del tiempo con proyectos que afectan al caudal del PHCCS.

Del análisis realizado de los tres proyectos, se desprende que del caudal del río Coca en el sector El Salado, se debería restar $20\left(\mathrm{~m}^{3} / \mathrm{s}\right)$ debido al caudal ecológico (ELC-Electroconsult, 2009); y en un futuro cercano el caudal de $4,06\left(\mathrm{~m}^{3} / \mathrm{s}\right)$ del proyecto CayambePedro Moncayo y Pesillo-Imbabura para el año 2018; un caudal de $2,21\left(\mathrm{~m}^{3} / \mathrm{s}\right)$ del proyecto Chalpi Grande, que corresponde a la etapa uno del PRO. Sin embargo, la concesión de 1987 , le permite utilizar hasta $5\left(\mathrm{~m}^{3} / \mathrm{s}\right)$, considerando que $3\left(\mathrm{~m}^{3} / \mathrm{s}\right)$ ya han sido utilizados en el proyecto Papallacta; y por último se debe considerar un total de $17,2\left(\mathrm{~m}^{3} / \mathrm{s}\right)$ del PRO correspondiente a la segunda y tercera etapa, que iniciarían en el año 2040 y 2041 respectivamente. En la Tabla 3 se explica más detalladamente los proyectos que restarían el caudal del PHCCS. Hay que considerar que la vida útil de un proyecto hidroeléctrico es generalmente de 50 a 75 años [39].

Como se observa en la Tabla 3, el caudal que ingresa al PHCCS disminuiría un valor máximo de 26,29 $\left(\mathrm{m}^{3} / \mathrm{s}\right)$, equivalente al $11 \%$ del caudal de diseño. Dado que el PHCCS es una central de pasada con regulación diaria, dicha disminución de caudal afectaría a la generación eléctrica en un porcentaje similar.
Tabla 3. Proyectos que influyen en el caudal del PHCCS

\begin{tabular}{ccc}
\hline Denominación & Año & Caudal $\left(\mathbf{m}^{3} / \mathbf{s}\right)$ \\
\hline Caudal ecológico & 2016 & 20 \\
Proyecto de riego & & \\
$\quad$ Cayambe-Pedro & 2018 & 4,06 \\
$\quad$ Moncayo y agua & & \\
potable Pesillo-Imbabura & & $\approx(2,21-5,00)$ \\
Proyecto Papallacta & 2021 & 17,23 \\
y PRO (etapa 1) & $2040-2055$ & $\approx(23,5-26,29)$ \\
PRO (etapa 2 y etapa 3) & &
\end{tabular}

\section{Conclusiones}

El Proyecto Hidroeléctrico Coca Codo Sinclair, vería afectada su capacidad de generación de energía por a la reducción del caudal de $222\left(\mathrm{~m}^{3} / \mathrm{s}\right)$ de entrada debido los futuros usos consuntivos del agua por parte de la EPMAPS, debido al Proyecto Chalpi Grande, que tomaría $2,2\left(\mathrm{~m}^{3} / \mathrm{s}\right)$ y las siguientes fases del Proyecto Ríos Orientales que tomarían hasta 17,2 $\left(\mathrm{m}^{3} / \mathrm{s}\right)$. A esto se restarían también los caudales utilizados por el Proyecto de riego Cayambe-Pedro Moncayo y el de agua potable Pesillo-Imbabura, que se tiene previsto culminar en el 2018, que en total tiene otorgada una concesión de 4,06 $\left(\mathrm{m}^{3} / \mathrm{s}\right)$. El caudal que ingresa al PHCCS se vería en el futuro reducido hasta en un 11 $\%$, afectando su generación eléctrica. Queda en evidencia la necesidad de planificar el aprovechamiento de los recursos considerando su nexo.

\section{Referencias}

[1] H. Hoff, "Understanding the nexus. background paper for the bonn2011 conference: The water, energy and food security nexus," in Stockholm Environment Institute, Stockholm, 2011. [Online]. Available: https://goo.gl/aiCG9J

[2] GWSP, "Sustainability in the water-energy-food nexus," in International Conference. Global Water System Project, 2014. [Online]. Available: https://goo.gl/CQjWTX

[3] OECD, Sustainable Management of Water Resources in Agriculture. Organisation for Economic Co-operation and Development, 2010. [Online]. Available: https: //doi.org/10.1787/9789264083578-en

[4] McKinsey\&Company, "Charting our water future. economic frameworks to inform decision-making," 2030 Water Resources Group, Tech. Rep., 2009. [Online]. Available: https://goo.gl/XEWfc3 
[5] WWAP (United Nations World Water Assessment Programme), "The united nations world water development report 2014: Water and energy," Paris, UNESCO., Tech. Rep., 2014. [Online]. Available: https://goo.gl/LFGg6d

[6] T. R. Albrecht, A. Crootof, and C. A. Scott, "The water-energy-food nexus: A systematic review of methods for nexus assessment," Environmental Research Letters, vol. 13, no. 4, pp. 1-26, 2018. [Online]. Available: https://doi.org/10.1088/1748-9326/aaa9c6

[7] C. Middleton, J. Allouche, D. Gyawali, and S. Allen, "The rise and implications of the waterenergy-food nexus in southeast asia through an environmental justice lens," Water Alternatives, vol. 8, no. 1, pp. 627-654, 2015. [Online]. Available: https://goo.gl/JKikGy

[8] H. Leck, D. Conway, M. Bradshaw, and J. Rees, "Tracing the water-energy-food nexus: Description, theory and practice," Geography Compass, vol. 9, no. 8, pp. 445-460, 2015. [Online]. Available: https://doi.org/10.1111/gec3.12222

[9] IEA, Water energy nexus. International Energy Agency. Secure Sustainable Together, 2016. [Online]. Available: https://goo.gl/DGsKrf

[10] D. Lofman, M. Petersen, and A. Bower, "Water, energy and environment nexus: The california experience," International Journal of Water Resources Development, vol. 18, no. 1, pp. 73-85, 2002. [Online]. Available: https://doi.org/10.1080/07900620220121666

[11] J. Pittock, D. Dumaresq, and A. M. Bassi, "Modeling the hydropower-food nexus in large river basins: A mekong case study," Water, vol. 8, no. 10, p. 425, 2016. [Online]. Available: https://doi.org/10.3390/w8100425

[12] F. Ackerman and J. Fisher, "Is there a waterenergy nexus in electricity generation? long-term scenarios for the western united states," Energy Policy, vol. 59, pp. 235-241, 2013. [Online]. Available: https://doi.org/10.1016/j.enpol.2013.03.027

[13] W. N. Lubega and A. M. Farid, "An engineering systems model for the quantitative analysis of the energy-water nexus," in Complex Systems Design \& Management, M. Aiguier, F. Boulanger, D. Krob, and C. Marchal, Eds. Cham: Springer International Publishing, 2014, pp. 219-231. [Online]. Available: https://doi.org/10.1007/978-3-319-02812-5_16

[14] U.S. Department of Energy, "The water-energy nexus: Challenges and opportunities," Tech. Rep., 2014. [Online]. Available: https://goo.gl/Q8ABT5
[15] Asamblea Constituyente, Capítulo quinto. Sectores estratégicos, servicios y empresas públicas. Art. 313. República del Ecuador, 2008. [Online]. Available: https://goo.gl/hFdWQm

[16] ENTRIX, Estudio de impacto ambiental preliminar del Proyecto Hidroeléctrico Coca Codo Sinclair. ENTRIX. Consultora Ambiental, 2008. [Online]. Available: https://goo.gl/zvSdEZ

[17] Asamblea Nacional, Ley Orgánica de Recursos Hidricos, Usos y Aprovechamiento del Agua. República del Ecuador. Secretaría del Agua, 2014. [Online]. Available: https://goo.gl/idEuHH

[18] Y. Granda Paladines, Estudio experimental en modelo hidraúlico fisico sobre la optimización de la bocatoma del Proyecto Coca-Codo-Sinclair. Tesis de Grado. Escuela Politécnica Nacional, 1992. [Online]. Available: https://goo.gl/q1RPsG

[19] V. López, "Implicaciones del proyecto Coca Codo Sinclair para la amazonía ecuatoriana," FLACSO, Tech. Rep., 2014. [Online]. Available: https://goo.gl/mP2PEx

[20] CENACE, Informe anual 201\%. Operador Nacional de Electricidad. Ministerio de Electricidad y Energía Renovable., 2017. [Online]. Available: https://goo.gl/Hc9wsB

[21] CELEC, Proyecto Hidroeléctrico Coca Codo Sinclair rediseño conceptual para 1500 MW. Corporación Eléctrica del Ecuador. Coca Codo Sinclair, 2009 .

[22] G. Rodríguez. (2014) El proyecto Coca Codo Sinclair: un lazo internacional hacia el desarrollo del Ecuador. Blog Ecuador hacia el desarrollo. [Online]. Available: https://goo.gl/sC2mkt

[23] A. Robles and I. Fernández, Centrales de generación de energía eléctrica. Universidad de Cantabria, 2012.

[24] Sinohydro Corporation, Contrato para el desarrollo de ingeniería para el Proyecto Hidroeléctrico Coca Codo Sinclair, 2009. [Online]. Available: https://goo.gl/JcQWea

[25] C. Mataix, Mecánica de fluidos y máquinas hidráulicas. Ediciones del Castillo S. A., 1986. [Online]. Available: https://goo.gl/4FZiEH

[26] J. E. Grijalva, Expansión y trayectorias de la ganadería en la Amazonía: estudio en el Valle de Quijos y Piedemonte, en Selva Alta. Ecuador, 2004. [Online]. Available: https://goo.gl/gf9vZL 
[27] INAMHI, Mapa de ubicación de la red hidrológica en operación por cuencas hidrográficas del Ecuador. Instituto Nacional de Meteorología e Hidrología. Ecuador., 2007. [Online]. Available: https://goo.gl/onJ7Qw

[28] GAD Papallacta, Plan de desarrollo y ordenamiento territorial Gobierno Autónomo Descentralizado parroquial de Papallacta, 2015. [Online]. Available: https://goo.gl/EvKSKM

[29] Secretaría del Agua, Resolución 201316-EPMAPS-Q., 2013. [Online]. Available: https://goo.gl/EhTFGG

[30] Ministerio del Ambiente, Plan de Manejo Adaptativo de la parte alta de la Reserva Ecológica Antisana. Ecuador, 2011. [Online]. Available: https://goo.gl/h1EXh1

[31] EPMAPS, Primera etapa del proyecto de agua potable ríos orientales ramal Chalpi Grande Papallacta. 2017. Empresa Pública Metropolitana de Agua Potable y Saneamiento. Alcaldía de Quito. Ecuador, 2017. [Online]. Available: https://goo.gl/D1RMZs

[32] ——, Estudios de factibilidad y diseños definitivos del proyecto de agua potable ramal Chalpi grande - Papallacta y central hidroeléctrica Chalpi grande. Empresa Pública Metropolitana de Agua Potable y Saneamiento. Alcaldía de Quito. Ecuador, 2014.
[33] Alcaldía de Quito, Quito tendrá abastecimiento de agua hasta el 2040 con el "Ramal Chalpi Grande-Papallacta", 2018. [Online]. Available: https://goo.gl/Be3Fg1

[34] L. Pachacama Oña and M. F. Cevallos López, Análisis de riesgo, vulnerabilidad de los estudios de la segunda etapa del proyecto de agua potable ríos orientales ramal Quijos-Papallacta-Paluguillo. Tesis de Grado. Escuela Politécnica del Ejército, 2012. [Online]. Available: https://goo.gl/rjrmWD

[35] IGM, Cartografía de Quito 1:25000. Instituto Geográfico Militar. Ecuador, 1990.

[36] Gestión de Comunicación. (2017) Sistema de Riego Cayambe-Pedro Moncayo. Prefectura de Pichincha. Ecuador. [Online]. Available: https://goo.gl/ReqZjP

[37] SENAGUA, Resolución de la Agencia de Aguas de Quito, proceso 220-96. Quito. Ecuador, 1999.

[38] SE, Resolución de la Agencia de Aguas de Quito, proceso 1375. Quito. Ecuador, 2005.

[39] T. Ochoa, Centrales hidroeléctricas tomo 1. Ediciones Grancolombianas. Universidad La Gran Colombia, 2002. 